\title{
FDG uptake, a surrogate of tumour hypoxia?
}

\author{
Rudi Andre Dierckx • Christophe Van de Wiele
}

Received: 23 October 2007 / Accepted: 18 February 2008 / Published online: 29 May 2008

(C) The Author(s) 2008

\begin{abstract}
Introduction Tumour hyperglycolysis is driven by activation of hypoxia-inducible factor-1 (HIF-1) through tumour hypoxia. Accordingly, the degree of 2-fluro-2-deoxy-Dglucose (FDG) uptake by tumours might indirectly reflect the level of hypoxia, obviating the need for more specific radiopharmaceuticals for hypoxia imaging.

Discussion In this paper, available data on the relationship between hypoxia and FDG uptake by tumour tissue in vitro and in vivo are reviewed. In pre-clinical in vitro studies, acute hypoxia was consistently shown to increase FDG uptake by normal and tumour cells within a couple of hours after onset with mobilisation or modification of glucose transporters optimising glucose uptake, followed by a delayed response with increased rates of transcription of GLUT mRNA. In pre-clinical imaging studies on chronic hypoxia that compared FDG uptake by tumours grown in rat or mice to uptake by FMISO, the pattern of normoxic and hypoxic regions within the human tumour xenografts, as imaged by FMISO, largely correlated with glucose metabolism although minor locoregional differences could not be excluded. In the clinical setting, data are limited and discordant.

Conclusion Further evaluation of FDG uptake by various tumour types in relation to intrinsic and bioreductive
\end{abstract}

R. A. Dierckx $(\triangle) \cdot$ C. Van de Wiele

Department of Nuclear Medicine and Molecular Imaging,

University Medical Center Groningen,

P.O. Box 30.001, 9700 RB Groningen, The Netherlands

e-mail: r.a.dierckx@ngmb.umcg.nl

C. Van de Wiele

e-mail: christophe.vandewiele@ugent.be

R. A. Dierckx $\cdot$ C. Van de Wiele

Department of Nuclear Medicine, University Hospital Ghent,

De Pintelaan 185 ,

9000 Ghent, Belgium markers of hypoxia and response to radiotherapy or hypoxia-dependent drugs is needed to fully assess its application as a marker of hypoxia in the clinical setting.

\section{Keywords FDG PET · Hypoxia}

\section{Introduction}

The most important non-surgical treatment for cancer is radiotherapy; of the $50 \%$ of cancer patients that can be currently cured, $70 \%$ receive radiation either as monotherapy or as part of a multimodality treatment. For a number of tumours such as squamous cell carcinoma of the head and neck or cervix carcinoma, radiotherapeutic treatment may fail due to the presence of tumour hypoxia $\left(\mathrm{pO}_{2}<5 \mathrm{mmHg}\right)$ which decreases the availability of oxygen free radicals that are necessary to induce sufficient DNA damage to cause cell death [1-4]. A non-invasive imaging technique allowing the identification and quantification of hypoxia in these patients may identify those patients that might benefit from concomitant radiosensitisation therapy or from dose escalation.

During the past two decades, several hypoxia-targeting radiopharmaceuticals have been developed. The chemical basis for most of these compounds, e.g. ${ }^{18} \mathrm{~F}$-misonidazole $\left({ }^{18} \mathrm{~F}\right.$-MISO) and $18 \mathrm{~F} / 123 \mathrm{I}$-iodoazomycin arabinoside $\left({ }^{18} \mathrm{~F}\right.$ AZA/ ${ }^{123}$ I-AZA, respectively), has been to incorporate a 2-nitroimidazole moiety to act as a bioreductive molecule accepting a single electron and producing a free radical anion, which, after further reduction, is then incorporated into cell constituents under hypoxic conditions $[5,6]$. For other non-imidazole moiety containing hypoxia-targeting radiopharmaceuticals, e.g. ${ }^{99 \mathrm{~m}} \mathrm{Tc}-2,2^{\prime}$-(1,4-diaminobutane) bis(2-methyl-3-butanone)dioxime $\left({ }^{99 \mathrm{~m}} \mathrm{Tc}-\mathrm{HL} 91\right)$ and ${ }^{62} \mathrm{Cu}-$ diacetyl-bis( $N$-4-methylthiosemicarbazone) $\quad\left({ }^{62} \mathrm{Cu}\right.$-ATSM), 
the mechanisms of tumour accumulation is not wellclarified, although it is generally accepted that the ${ }^{99 \mathrm{~m}} \mathrm{Tc}$ and ${ }^{62} \mathrm{Cu}$ complexes are bioreducible groups themselves. To find routine clinical application, these radiopharmaceuticals should be widely available, provide meaningful averages over tumour volumes and allow for administration, before, during and after the investigation of tumour oxygenation and re-oxygenation. Unfortunately, their synthesis, however, requires either the presence of a cyclotron or specialised in-house radiopharmacy expertise, facilities still unavailable to most nuclear medicine departments $[5$, 6]. Contrary to hypoxia-targeting radiopharmaceuticals, 2fluro-2-deoxy-D-glucose (FDG) is widely available. The basis for the application of FDG PET imaging in oncology is the upregulation of glucose transporters (GLUTs) and glycolytic enzymes also known as the Warburg effect or tumour hyperglycolysis $[7,8]$. It is important to note that tumour hyperglycolysis is driven by the activation of hypoxia-inducible factor-1 (HIF-1) either through genetic alterations or changes of levels of growth factors and also via tumour hypoxia. Accordingly, the degree of FDG uptake by tumours might indirectly reflect the level of hypoxia, obviating the need for more specific radiopharmaceuticals for hypoxia imaging. This paper reviews available data on the relationship between hypoxia and FDG uptake by tumour tissue in vitro and in vivo.

Pre-clinical findings

\section{Cell line findings}

The uptake of ${ }^{3} \mathrm{H}-\mathrm{FDG}$ into two human tumour cell lines (HTB 63 melanoma and HTB 77 IP3 ovarian carcinoma) was determined after exposure to differing oxygen atmospheres ranging from $0 \%$ to $20 \% \mathrm{O}_{2}$ for varying time periods by Clavo et al. [9]. FDG uptake in both melanoma and ovarian carcinoma cell lines increased significantly $(39.6 \pm 6.7 \%$ and $36.7 \pm 9 \%$, respectively) over basal $(20 \%$ $\mathrm{O}_{2}$ ) conditions when cells were exposed to a mild hypoxic environment $\left(5 \% \mathrm{O}_{2}\right)$ for $1.5 \mathrm{~h}$. With a 4-h exposure to $1.5 \% \mathrm{O}_{2}$, the increase in FDG uptake was greater at $52.3 \pm$ $8.9 \%$ and $43.5 \pm 19 \%$, respectively. With $4 \mathrm{~h}$ of anoxia, the increase in FDG uptake over basal conditions was $42.7 \pm 10 \%$ and $63.3 \pm 13.7 \%$ for melanoma and ovarian carcinoma cells, respectively. Membrane transport of 3-O-methylglucose (3-OMG) was increased by hypoxia for melanoma and ovarian carcinoma. Immunochemical assays for Glut-1 showed an increase in the membrane expression of the Glut-1 transporter in cells exposed to hypoxia.

Hypoxic accumulation of 2-[5,6-3H]fluoro-2-deoxy-Dglucose was evaluated in two cell lines (UT-SCC-5 and UTSCC-20) obtained from patients with squamous cell carcinoma of the head and neck by Minn et al. [10]. Both cell lines were exposed to decreasing oxygen atmosphere $\left(20 \%, 1.5 \%\right.$, or $\left.0 \% \mathrm{O}_{2}\right)$ for $6 \mathrm{~h}$, after which they were incubated for a further $1 \mathrm{~h}$ with tritiated FDG. An anoxic atmosphere resulted in a mean increase of ${ }^{3} \mathrm{H}$-FDG uptake of $120 \%$ and $46 \%$ over a baseline $20 \%$ oxygen atmosphere for UT-SCC-5 and UT-SCC-20A, respectively.

Similarly, a more than twofold increase $(2.53 \pm 0.79 ; p<$ $0.005)$ in ${ }^{3} \mathrm{H}-\mathrm{FDG}$ uptake was observed under hypoxic conditions by Burgman et al. by MCF-7 breast cancer cells, but no changes in the cellular levels of glucose transporter proteins (GLUT1 and GLUT3) or hexokinase were observed [11]. A reducing agent, dithiothreitol (DTT), also caused an increase in ${ }^{3} \mathrm{H}-\mathrm{FDG}$ uptake but failed to affect uptake under hypoxic conditions. This indicates that the mechanisms by which hypoxia and DTT affect ${ }^{3} \mathrm{H}-\mathrm{FDG}$ uptake might be the same. The oxidising agent chloromercuribenzenesulphonic acid (pCMBS) had no effect on ${ }^{3} \mathrm{H}$ FDG uptake under normoxic conditions but counteracted the effect of hypoxia. DTT caused an increase in glucose transporter activity, whereas it had no effect on hexokinase activity. pCMBS had no effect on either glucose transporter activity or hexokinase activity. The authors concluded that hypoxia-induced increase in ${ }^{3} \mathrm{H}-\mathrm{FDG}$ uptake in MCF7 cells is the result, in part, of an increase in glucose transporter activity resulting from the modification (reduction) of thiol group(s) in the glucose transport protein(s) and that the modulation of hexokinase activity is probably not involved in the hypoxia-induced increase in ${ }^{3} \mathrm{H}-\mathrm{FDG}$ uptake in these cells.

Pedersen et al. studied the relationship between ${ }^{18} \mathrm{~F}$ labelled FDG uptake and expression of glucose transporters (GLUTs 1, 2 and 3) in two human small cell lung cancer (SCLC) lines CPH 54A and CPH 54B [12]. Changes in the expression of GLUTs and vascular endothelial growth factor (VEGF) during 12, 18, and $24 \mathrm{~h}$ of severe hypoxia in vivo (xenografts) and in vitro (cell cultures) were recorded for both tumour lines. There were higher levels of Glut-1 protein in 54B and a correspondingly higher FDG uptake in this tumour line $(p<0.001)$. During hypoxia, a significant upregulation of VEGF mRNA, GLUT-1 mRNA and Glut-1 and Glut-3 proteins occurred with a distinctly different time course in the two cell lines. A similar coupregulation of GLUT and VEGF was seen in hypoxic tumours of both lines. There were no significant changes of HIF-1alpha mRNA during hypoxia in either of the cell lines.

Hara et al. monitored the uptake of $\left[{ }^{18} \mathrm{~F}\right]$ 2-fluoro-2deoxyglucose $\left({ }^{18} \mathrm{~F}\right.$-FDG) in androgen-independent $\mathrm{PC}-3$ cells and androgen-sensitive LNCaP cells under aerobic or anoxic conditions [13]. The effect of androgen depletion was also examined. After $4 \mathrm{~h}$ of anoxia, PC-3 cells showed an $85 \%$ increase in FDG uptake whereas $\mathrm{LNCaP}$ cells showed a $212 \%$ increase in FDG uptake. Of interest, 
androgen depletion resulted in a marked decrease in the uptake of ${ }^{18} \mathrm{~F}$-FDG in LNCaP cells but not in PC-3 cells.

Finally, Oswald et al. studied FDG uptake in three tumour cell lines, namely, HT-29 (colorectal), FaDu (SCCHN) and MCF-7 (HT-29 and FaDu) and in three primary endothelial cell lines cultured at normoxic and hypoxic conditions [14]. In comparison to normoxic conditions, cellular uptake of ${ }^{18} \mathrm{~F}-\mathrm{FDG}$ was significantly increased at hypoxic conditions in two of the three tumour cell lines under study and all three endothelial cell lines.

\section{Animal models}

Dearling et al. injected mice (MF1) bearing LS174T human tumour xenografts premixed ${ }^{18} \mathrm{~F}$-FDG $(100 \mathrm{MBq}),{ }^{14} \mathrm{C}$ $2 \mathrm{DG}(0.37 \mathrm{MBq})$ and pimonidazole hydrochloride $(60 \mathrm{mg} / \mathrm{kg})$ [15]. After 30, 60 and $120 \mathrm{~min}$, tissues $(n=4)$ were taken and counted for whole-body biodistribution. Tumours were frozen, sectioned and exposed to phosphor image plates to obtain a quantitative digital image of radionuclide distribution. Sections were then stained to reveal tumour pathophysiology. Viable tissues were separated from necrotic tissues based on haematoxylin and eosin staining and manually based on regions of interest. Within these regions, the amount of hypoxic (brown staining or pimonidazole-positive) and normoxic tissue (blue staining or pimonidazole-negative) was assessed using an automated colour-discriminating in-house written software programme. Whole-body biodistributions and tumour microdistribution for the tracers were similar $(r=0.85 \pm 0.10, p<0.0001$ for tumour uptake). When the tumours were divided into viable and necrotic regions, the ratio of mean counts per pixel was $1.96(p<0.0001)$, whereas for hypoxic versus normoxic regions, it was $1.26(p<0.0001)$. There was no significant difference in selectivity for hypoxia between the tracers $(p=$ 0.86 ). This study confirmed the selectivity of deoxyglucose for viable cells over necrotic regions and for hypoxic cells over normoxic regions in vivo.

FDG PET and ${ }^{18} \mathrm{~F}$-FMISO PET imaging was performed with a dedicated small-animal PET scanner NanoPET (Oxford Positron Systems) in ten different human tumour xenografts in nude mice and B16 melanoma tumours in syngeneic Balb/c mice by Wyss et al. The pattern of normoxic and hypoxic regions within the human tumour xenografts, as imaged by ${ }^{18} \mathrm{~F}$-FMISO and ${ }^{18} \mathrm{~F}$-FDG proved similar. In addition, the uptake values of FDG and parameters used to quantify FMISO accumulation exhibited a strong positive correlation [16].

Zanzonico et al. studied the intra-tumoural distributions of sequentially administered F18-fluoro-deoxyglucose (FDG) and the hypoxia tracer ${ }^{18} \mathrm{~F}$-fluoromisonidazole (FMISO) were compared in nude rats with a hindlimb
R3327-AT anaplastic rat prostate tumour xenograft and a hindlimb FaDu human squamous cell carcinoma (each up to $20 \times 20 \times 30 \mathrm{~mm}$ in size) rats by registered micro-PET imaging with positioning of each animal in a customfabricated whole-body mould [17]. The registered microPET images unambiguously demonstrated grossly similar although not identical distributions of FDG and FMISO in the tumours-a high-activity rim surrounding a loweractivity core. There were, however, subtle but possibly significant differences in the intra-tumoural distributions of FDG and FMISO.

Tanaka et al. studied the intra-tumoural distribution of ${ }^{64} \mathrm{Cu}$-diacetyl-bis(N4-methylthiosemicarbazone) $\left(\left[{ }^{64} \mathrm{Cu}\right] \mathrm{Cu}\right.$ ATSM) and ${ }^{18}$ F-FDG in mice with tumours of four different origins (LLC1, Meth-A, B16 and colon26) compared to immunohistochemical staining of proliferating cells (Ki67), blood vessels (CD34 or von Willebrand factor), and apoptotic cells (terminal deoxynucleotidyltransferasemediated dUTP nick end labelling method) [18]. With all cell lines, $\left[{ }^{64} \mathrm{Cu}\right] \mathrm{Cu}$-ATSM and ${ }^{18} \mathrm{~F}$-FDG were distributed with different gradation in the tumour mass. The immunohistochemical study suggested that the high $\left[{ }^{64} \mathrm{Cu}\right] \mathrm{Cu}$ ATSM uptake regions were hypovascular and consisted of tumour cells arrested in the cell cycle, whereas the high ${ }^{18} \mathrm{~F}$-FDG uptake regions were hypervascular and consisted of proliferating cells.

\section{Clinical findings}

Squamous cell carcinoma of the head and neck (SCCHN)

Zimny et al. studied 24 patients suffering from SCCHN with metastatic lymph nodes that underwent FDG PET, FMISO PET and $\mathrm{pO}_{2}$-polarography within 1 week [19]. Parameters of $\mathrm{pO}_{2}$-polarography were the relative frequency of $\mathrm{pO}_{2}$ readings $\leq 2.5, \leq 5$ and $\leq 10 \mathrm{mmHg}$, respectively, and the mean and median $\mathrm{pO}_{2}$ in lymph nodes under study. Whilst a moderate correlation of the maximum standardised uptake value (SUVmax) of FDG with the tumour and/or lymph node to blood ratio of FMISO at $2 \mathrm{~h}(r=0.53, p<$ 0.05 ) was observed, SUV of FDG was similar in hypoxic and normoxic tumours as defined by $\mathrm{pO}_{2}$-polarography $(6.9 \pm 3.2$ vs $6.2 \pm 3.0$, NS) and the FDG uptake was not correlated with the results of $\mathrm{pO}_{2}$-polarography whereas FMISO uptake was $(r=0.80, p<0.001)$ [19]. These results were later on confirmed by the same authors in an extended series of 36 patients [20].

Thorwarth et al. studied 12 patients suffering from advanced head and neck cancer (HNC) that underwent FDG and FMISO PET examinations before RT treatment. In this series, tumour volume was defined in two steps; first through region growing, using a threshold of $60 \%$ of the maximum FDG uptake value followed by an extension of 
this volume by 3 voxels in each direction yielding the final tumour volume [21]. The percentage of voxels of each tumour that presented FMISO SUV values at $2 \mathrm{~h}$ pi. larger than 1.4 defined the hypoxic fraction of each tumour. The authors also performed a scatter-plot based voxel-by-voxel FDG-FMISO correlation for each patient for the defined tumour volumes and assessed whether the slope of the regression line $(\mathrm{m})$ and the scatter width of the scatter plots (sigma) allowed to stratify the patient group with respect to treatment outcome. No general correlation of enhanced glucose metabolism and FMISO uptake was observed. In seven out of the 12 patients studied, scatter patterns for the primary tumour were very narrow suggesting a functional dependence of FDG and FMISO uptake. In the two patients in whom lymph nodes were also included for analysis, widely dispersed data points of FDG and FMISO SUVs were found for lymph nodes. Finally, whereas the maximum uptake of FMISO $(p=0.045)$ showed borderline significance for stratifying the patient group, FDG positive tumour volume, hypoxic fraction, maximum FDG uptake and $m$ were not significant. Sigma turned out to be the most significant variable $(p=0.008)$ to predict treatment success probabilities.

In a study by Rajendran et al., the mean correlation coefficient between FMISO and FDG uptake by primary SCCHN on a pixel-by-pixel basis per tumour was 0.62 [22]. In this study, patients suffering from different $\mathrm{T}$-stages were included and FDG uptake by the primary tumour covered a wide range (FDG SUV $\max =2.9-25.4$ ).

Non-small cell lung carcinoma (NSCLC)

Cherk et al. prospectively studied 21 patients with suspected or biopsy-proven NSCLC that underwent both FMISO and FDG PET [23]. Seventeen patients subsequently underwent surgery with analysis performed for tumour markers of angiogenesis and hypoxia. In the 17 patients with resectable NSCLC (13 men, 4 women; age range $=51-77$ years), the mean FMISO uptake in tumour was significantly lower than that of FDG uptake $(p<0.0001)$ and showed no correlation with FDG uptake $(r=0.26)$. The hypoxic cell fraction of primary NSCLC was consistently low, and there was no significant correlation in NSCLC between hypoxia and glucose metabolism in NSCLC assessed by ${ }^{18}$ F-FDG.

Similar findings were obtained by Gagel et al. in a series of 18 NSCLC patients who also underwent both FMISO and FDG PET [24].

Finally, Dehdashti et al. performed ${ }^{60} \mathrm{Cu}$-ATSM and FDG PET in a series of 19 NSCLC carcinoma patients. In the 14 patients in whom response to therapy was assessed, no significant correlation between tumour ${ }^{60} \mathrm{Cu}$-ATSM and FDG SUV max values $(r=0.04 ; p=0.9)$ was found [25].
Other tumour types

Cher et al. studied 17 patients with suspected primary glioma with ${ }^{18} \mathrm{~F}$-FMISO and ${ }^{18} \mathrm{~F}$-FDG PET [26]. There was $100 \%$ concordance as to the absence or presence of uptake of both tracers in all tumours under study with five patients displaying a lack of uptake of either tracer preferentially in low-grade tumours. The pattern of uptake of both tracers within positive tumours was, however, different with areas of marked FMISO uptake often corresponding to areas of only slightly increased FDG uptake.

Finally, no significant correlation was found between FDG SUV max values and FMISO SUVmax values in patients suffering from soft tissue sarcoma, breast cancer or glioblastoma multiforme ( $r$ values, respectively, $0.47,0.38$ and 0.32 ; number of patients studied, respectively, 11, 7 and 5) [22].

\section{Discussion}

Solid tumours consist of regions with large numbers of hypoxic cells compared with the surrounding normal tissue with substantial heterogeneity within a given tumour and among tumours of the same histology [7, 8]. The underlying process involved in generating a hypoxic micro-environment is not well-understood but likely include various mechanisms of impaired intra-tumoural oxygen delivery and consumption. In particular, tumour hypoxia seems to arise from the stochastic development of vasculature during tumour growth. Tumour blood vessels are highly irregular, lack smooth muscles and innervation, may have an incomplete endothelial lining and basement membrane and often develop from post capillary venules, creating severe longitudinal $\mathrm{pO}_{2}$ gradients within the vessels themselves. Because of these factors, a significant proportion of tumour cells lie beyond the diffusion distance of oxygen, rendering them chronically or diffusion-limited hypoxic. In addition, tumour hypoxia can also occur acutely because of transient occlusion, narrowing of vessels, arteriolar vasomotion and variation in red cell traffic patterns. Models based on data generated from experimental studies suggest that substantial volumes of solid tumours are exposed to cyclic episodes of acute or transient hypoxia. Both acute and chronic hypoxia are present at any time in tumour tissues, the latter form predominating, and their influence on FDG uptake has been studied by various authors.

In pre-clinical in vitro studies, acute hypoxia was consistently shown to increase FDG uptake by normal and tumour cells within a couple of hours after onset [9-13]. The time course and extent of uptake proved very different from one tumour cell line to another. In addition, in those 
few studies that looked at associated changes in glucose transporter expression and hexokinase activity, a significant increase in the number of glucose transporters but not hexokinase activity was evidenced which may be due to recycling of internalised receptors to the cell membrane or to a decreased rate of internalisation of GLUTs. In addition, in one study by Burgman et al., it was suggested that modification (reduction) of thiol group(s) present on GLUTs through hypoxia may increase the kinetics of glucose transport by GLUTs [11]. In the one study that performed delayed assessment of glucose uptake after 18$24 \mathrm{~h}$ of acute hypoxia, mRNA analysis was performed revealing increased levels of mRNA of amongst others GLUTs and VEGF [12]. Overall, these findings suggest a rapid onset response of cells to hypoxia with mobilisation or modification of GLUTs optimising glucose uptake, followed by a delayed response with increased rates of transcription of GLUT mRNA.

In pre-clinical imaging studies on chronic hypoxia that compared FDG uptake by tumours grown in rats or mice to uptake by FMISO, the pattern of normoxic and hypoxic regions within the human tumour xenografts, as imaged by FMISO, largely correlated with glucose metabolism although minor locoregional differences could not be excluded $[16,17]$. This finding suggests a compensatory mechanism for the reduced delivery of glucose to hypoxic tumour cells by augmented tissue extraction owing to elevated glucose transporter activity under hypoxia in the tumour models under study. As opposed to FMISO, in the one study that compared ${ }^{64} \mathrm{Cu}$-ASTM to FDG uptake by tumour tissues, findings proved discordant [18]. In all four tumour models under study, LLC1, colon26, Meth-A and B16, FGD and ${ }^{64} \mathrm{Cu}$-ATSM were accumulated with different gradation forming a unique pattern of a high ${ }^{64} \mathrm{Cu}-\mathrm{ATSM}$ region surrounding a high FDG region within a tumour mass which in turn surrounded a necrotic core. The imaging results obtained with ${ }^{64} \mathrm{Cu}$-ATSM are at the least to say surprising and quite opposite to the general model of tumour hypoxia that includes a peripheral viable core, a central necrotic core and a hypoxia rim in between. Contrariwise, the images obtained with FDG proved similar to those obtained by Dearling et al. who compared pimonidazole staining with FDG uptake in a MCF-7 tumour model and found similar results for both tracers [15]. Due to the lack of a gold standard for hypoxia, e.g. polarography, no definitive conclusions can be derived from this study.

Data reporting on the relationship between FDG uptake and tumour hypoxia in the clinical setting are limited. In patients suffering from squamous cell carcinoma of the head and neck, studies have looked at the relationship between hypoxia and FDG uptake in both lymph nodes (one cumulative series of 36 patients by Gagel et al.) and primary tumours (two series of patients suffering from SCCHN, respectively, one on 12 patients by Thorwarth et al. and one reporting on 38 patients by Rajendran et al.) with seemingly conflicting results [19-22]. Whereas no relationship was found between FDG uptake and hypoxia in lymph nodes and in the smaller study in primary tumours by Thorwarth et al., a significant relationship was found in primary tumours in the study by Rajendran et al. [21, 22]. Based on available data, hypoxic regions appear to be distributed homogeneously in cervical lymph node metastases from SCCHN and there is no gradient of oxygenation, contrary to what is seen in primary tumours [27]. In addition, the level of hypoxia is inversely correlated with lymph node perfusion, suggesting that hypoxia in invaded lymph nodes is flow-related rather than diffusion-related as is the case in primary tumours [27]. It is currently unknown whether the degree of hypoxia is also related to the level of lymph node involvement or not. The lack of such a relationship might also explain the lack of a relationship between hypoxia and FDG accumulation in lymph nodes as demonstrated in the series by Gagel et al. In the series by Thorwarth et al., a selective group of patients appears to have been included based on the narrow range of FDG SUVmax values (from 7.84 to 12.07 ; mean $=9.53$ ) of the primary tumour. Within this narrow range, a much larger cohort of patients is likely required to prove or exclude the existence of a relationship between FDG and FMISO uptake. Opposite to the series by Thorwarth et al., in the study by Rajendran et al. that did find a relationship between FDG and FMISO accumulation, FDG SUV values covered a wide range (from 2.9 to 25.4 ; mean=10.9).

In the few studies that assessed FDG uptake versus FMISO uptake in a limited number of patients suffering from NSCLC, glioblastoma multiforme or breast cancer, no correlation was found between both variables [22-24].

Based on recent data, it appears that HIF-1 expression may also be observed in non-hypoxic tumour regions, suggesting that not only the hypoxic micro-environment but also non-hypoxic factors such as genetic alterations or levels of growth factors affect HIF-1 levels and thus also indirectly affect glucose metabolism [7, 8]. Accordingly, it cannot be excluded that the existence of a correlation or lack of correlation between hypoxia and FDG accumulation is tumour type-dependent.

To conclude, further evaluation of FDG uptake by various tumour types in relation to intrinsic and bioreductive markers of hypoxia and response to radiotherapy or hypoxia-dependent drugs is needed to fully assess its role as a marker of hypoxia in the clinical setting. In particular, the relationship between HIF-1 activation and hypoxia in various human malignancies warrants further study. Whilst from a biological point of view, FDG uptake by tumour cells reflects a different aspect of tumour biology when 
compared to the uptake of e.g. FMISO or other hypoxiatargeting radiopharmaceuticals in those tumours where HIF-1 activation is mainly hypoxia-driven, the degree of FDG uptake may be a surrogate marker of hypoxia. Inversely, in those tumours where HIF-1 activation occurs mainly independently of hypoxia, specific hypoxia-targeting radiopharmaceuticals such as FMISO or ${ }^{64} \mathrm{Cu}-\mathrm{ATSM}$ may be required to non-invasively image tumour hypoxia.

Open Access This article is distributed under the terms of the Creative Commons Attribution Noncommercial License which permits any noncommercial use, distribution, and reproduction in any medium, provided the original author(s) and source are credited.

\section{References}

1. Fyles AW, Milosevic M, Wong R, Kavanagh M, Pintilie M, Sun A, et al. Oxygenation predicts radiation response and survival in patients with cervix cancer. Radiother Oncol 1998;48:149-56.

2. Hockel M, Schlenger K, Hockel S. Association between tumor hypoxia and malignant progression: the clinical evidence in cancer of the uterine cervix. In: Vaupel P, Kelleher DK, editors. Tumour hypoxia. Stuttgart: Wissenschafte Verlagsgesellschaft mbH; 1999. p. $65-74$.

3. Nordsmark M, Overgaard M, Overgaard J. Pretreatment oxygenation predicts radiation response in advanced squamous cell carcinoma of the head and neck. Radiother Oncol 1996;41:31-9.

4. Stone HB, Brown JM, Philips TL, Suherland R. Oxygen in human tumors: correlations between methods of measurement and response to therapy. Summary of a workshop held November 19-20, 1992, at the National Cancer Institute, Bethesda, MD. Radiat Res 1993;136:422-34.

5. Padhani A, Krohn K, Lewis J, Alber M. Imaging oxygenation of human tumours. Eur Radiol 2007;17:861-72.

6. Van de Wiele C, Lahorte C, Oyen W, Boerman O, Goethals I, Slegers G, Dierckx RA. Nuclear medicine imaging to predict response to radiotherapy: a review. Int J Radiat Oncol Biol Phys 2003;55:5-15.

7. Airley RE, Mobasheri A. Hypoxic regulation of glucose transport, anaerobic metabolism and angiogenesis in cancer: novel pathways and targets of anticancer therapeutics. Chemotherapy 2007;53:233-56.

8. Kim JW, Gao P, Dang C. Effects of hypoxia on tumor metabolism. Cancer Metastasis Rev 2007;26:291-98.

9. Clavo AC, Brown RS, Wahl RL. Fluorodeoxyglucose uptake in human cancer cell lines is increased by hypoxia. J Nucl Med 1995;36:1625-32.

10. Minn H, Clavo AC, Wahl RL. Influence of hypoxia on tracer accumulation in squamous-cell carcinoma: in vitro evaluation for PET imaging. Nucl Med Biol 1996;23:941-46.

11. Burgman P, Odonoghue JA, Humm JL, Ling CC. Hypoxiainduced increase in FDG uptake in MCF7 cells. J Nucl Med 2001;42:170-5.

12. Pedersen MW, Holm S, Lund EL, Hojgaard L, Kristjansen PE. Coregulation of glucose uptake and vascular endothelial growth factor (VEGF) in two small-cell lung cancer (SCLC) sublines in vivo and in vitro. Neoplasia 2001;3:80-7.

13. Hara T, Bansal A, DeGrado TR. Effect of hypoxia on the uptake of [methyl-3H]choline, $[1-14 \mathrm{C}]$ acetate and [18F]FDG in cultured prostate cancer cells. Nucl Med Biol 2006;33:977-84.
14. Oswald J, Treite F, Haase C, Kampfrath T, Mäding P, Schwenzer $\mathrm{B}$, et al. Experimental hypoxia is a potent stimulus for radiotracer uptake in vitro: comparison of different tumor cells and primary endothelial cells. Cancer Lett 2007 28;254:102-10.

15. Dearling J, Flynn A, Sutcliffe-Goulden J, Petrie L, Boden R, Green A, et al. Analysis of the regional uptake of radiolabeled deoxyglucose analogs in human tumor xenografts. J Nucl Med 2004;45:101-7.

16. Wyss MT, Honer M, Schubiger PA, Ametamey SM. NanoPET imaging of $[(18)$ F] fluoromisonidazole uptake in experimental mouse tumours. Eur J Nucl Med Mol Imaging 2006;33:311-18.

17. Zanzonico P, Campa J, Polycarpe-Holman D, Forster G, Finn R, Larson S, et al. Animal-specific positioning molds for registration of repeat imaging studies: comparative microPET imaging of F18labeled fluoro-deoxyglucose and fluoro-misonidazole in rodent tumors. Nucl Med Biol 2006;33:65-70.

18. Tanaka T, Furukawa T, Fujieda S, Kasamatsu S, Yonekura Y, Fujibayashi Y. Double-tracer autoradiography with $\mathrm{Cu}$-ATSM/FDG and immunohistochemical interpretation in four different mouse implanted tumor models. Nucl Med Biol 2006;33(6):743-50.

19. Zimny $M$, Gagel B, DiMartino $E$, Hamacher $K$, Coenen $H$, Westhofen M, et al. FDG-a marker of tumour hypoxia? A comparison with [(18)F]fluoromisonidazole and $\mathrm{pO}_{2}$-polarography in metastatic head and neck cancer. Eur J Nucl Med Mol Imaging 2006;33:1426-31.

20. Gagel B, Piroth M, Pinkawa M, Reinartz P, Zimny M, Kaiser HJ, et al. $\mathrm{pO}_{2}$ polarography, contrast enhanced color duplex sonography $(\mathrm{CDS}),[18 \mathrm{~F}]$ fluoromisonidazole and $[18 \mathrm{~F}]$ fluorodeoxyglucose positron emission tomography: validated methods for the evaluation of therapy-relevant tumor oxygenation or only bricks in the puzzle of tumor hypoxia? BMC Cancer 2007;7:113. DOI 10.1186/1471-2470-7-113, Jun 28.

21. Thorwarth D, Eschmann SM, Holzner F, Paulsen F, Alber M. Combined uptake of [18F]FDG and [18F]FMISO correlates with radiation therapy outcome in head-and-neck cancer patients. Radiother Oncol 2006;80:151-56.

22. Rajendran JG, Mankoff DA, O'Sullivan F, Peterson LM, Schwartz DV, Conrad EV, et al. Hypoxia and glucose metabolism in malignant tumors: evaluation by $\left.{ }^{18} \mathrm{~F}\right]$ fluoromisonidazole and $\left[{ }^{18} \mathrm{~F}\right]$ fluorodeoxyglucose positron emission tomography. Clin Cancer Res 2004;10:2245-52.

23. Cherk MH, Foo SS, Poon AM, Knight SR, Murone C, Papenfuss AT, et al. Lack of correlation of hypoxic cell fraction and angiogenesis with glucose metabolic rate in non-small cell lung cancer assessed by 18 F-Fluoromisonidazole and 18F-FDG PET. J Nucl Med 2006;47:1921.

24. Gagel B, Reinartz P, Demirel C, Kaiser HJ, Zimny M, Piroth M, et al. [18F] fluoromisonidazole and $[18 \mathrm{~F}]$ fluorodeoxyglucose positron emission tomography in response evaluation after chemo-/radiotherapy of non-small-cell lung cancer: a feasibility study. BMC Cancer 2004;4(6):51.

25. Dehdashti F, Mintun MA, Lewis JS, Bradley J, Govindan R, Laforest $\mathrm{R}$, et al. In vivo assessment of tumor hypoxia in lung cancer with $60 \mathrm{Cu}$-ATSM. Eur J Nucl Med Mol Imaging 2003;30:844-50.

26. Cher L, Murone C, Lawrentschuk N, Ramdave S, Papenfusse A, Hannah A, et al. Correlation of hypoxic cell fraction and angiogenesis with glucose metabolic rate in gliomas using $18 \mathrm{~F}$ fluoromisonidazole, 18F-FDG PET, and immunohistochemical studies. J Nucl Med 2006;47:410-18.

27. Scholbach T, Scholbach J, Krombach GA, Gagel B, Maneschi P, Di Martino E. New method of dynamic color doppler signal quantification in metastatic lymph nodes compared to direct polarographic measurements of tissue oxygenation. Int $\mathrm{J}$ Cancer 2005;114:957-62. 